\title{
Maneuvering Target Tracking Algorithm Based on Interacting Multiple Models
}

\author{
Gannan Yuan, Wei Zhu, Wei Wang, and Bo Yin \\ College of Automation, Harbin Engineering University, No. 145, Nantong Street, Harbin 150001, China \\ Correspondence should be addressed to Wei Zhu; zhuwei_heu@163.com
}

Received 2 January 2015; Revised 23 April 2015; Accepted 25 April 2015

Academic Editor: Erik Cuevas

Copyright ( 2015 Gannan Yuan et al. This is an open access article distributed under the Creative Commons Attribution License, which permits unrestricted use, distribution, and reproduction in any medium, provided the original work is properly cited.

\begin{abstract}
Aiming at improving the accuracy and quick response of the filter in nonlinear maneuvering target tracking problems, the Interacting Multiple Models Cubature Information Filter (IMMCIF) is proposed. In IMMCIF, the Cubature Information Filter (CIF) is brought into Interacting Multiple Model (IMM), which can not only improve the accuracy but also enhance the quick response of the filter. CIF is a multisensor nonlinear filtering algorithm; it evaluates the information vector and information matrix rather than state vector and covariance, which can reduce the error of nonlinear filtering algorithm. IMM disposes all the models simultaneously through Markov Chain, which can enhance the quick response of the filter. Finally, the simulation results show that the proposed filter exhibits fast and smooth switching when disposing different maneuver models; it performs better than the IMMCKF and IMMUKF on tracking accuracy.
\end{abstract}

\section{Introduction}

Nowadays, target tracking has broad application in different areas, such as radar tracking, aircraft surveillance, and vehicle navigation [1-4]. In order to improve the tracking precision when the motion of the target is complex, many filtering algorithms have been proposed based on specifically physical model [5-7]. Blom and Bar-Shalom propose the IMM algorithm based on generalized pseudorandom algorithm to depress the error of the single model algorithm [8]. The IMM algorithm processes all the models simultaneously and then switches to different models by checking the weight of each filter [9]. Analysis shows that IMM algorithm is more robust and more exact than the single model algorithm when tracking the maneuvering target $[10,11]$.

In order to enhance the performance of IMM algorithm, many nonlinear filters have been designed. For example, Interacting Multiple Models Extended Kalman Filter (IMMEKF) and Interacting Multiple Models Unscented Kalman Filter (IMMUKF) $[4,10,12-14]$ are proposed as IMM-based nonlinear filtering algorithm. The results show that, in maneuvering targets tracking problems, IMMUKF performs better than IMMEKF in stability and precision [11]. It is because
Extended Kalman Filter (EKF) carries on the filtering procession by linearizing the state equation, which inevitably induces the linearization error even divergence of filter. However, when Unscented Kalman Filter (UKF) [15] is used in higher order system, the weights of the sigma points will turn into negative which will result in the divergence of the filter. In order to overcome these issues and improve the performance of filter, Cubature Kalman Filter (CKF) is introduced in IMM to track the maneuvering target $[14,16]$. And analysis shows that the IMMCKF exhibits better performance than IMMUKF and IMMEKF in enhancing the accuracy and reducing the computational complexity [14]. In this paper, the Interacting Multiple Models Cubature Information Filter (IMMCIF) based on IMM and the CIF $[17,18]$ is proposed to improve the tracking accuracy and quick response of maneuvering target. In IMMCIF, the state estimation variables including position and velocity of different models are disposed simultaneously through Markov Chain; then the combined state estimate and covariance of each filter are fed back when the cycle completes. The proposed filter evaluates the information vector and information matrix instead of the state vector and covariance; it propagates in the information space which makes the filter easy to initialize and more 
precise [17]. Therefore, the accuracy of the IMM is improved, and the novel algorithm in this paper is a promising approach for maneuvering target tracking. The numerical simulation results show that the IMMCIF exhibits better than IMMCKF and IMMUKF on accuracy, robustness, and switching response.

The remainder of this paper is organized as follows. In Section 2, the Cubature Information Filter is briefly reviewed. The whole procession of IMMCIF used in target tracking problem is derived in Section 3. In Section 4, the performance of IMMCIF is better than IMMCKF and IMMUKF, they are compared in a benchmarked target tracking problem, and the results are analyzed. Conclusions are given in Section 5 .

\section{Cubature Information Filter}

The CIF is different with conventional filtering algorithms which are based on Kalman filter. It estimates information state vector and information matrix instead of state vector and covariance matrix, from which the measurement update is simpler than CKF.

\subsection{Time Update. Consider}

$$
\left[Y_{k-1 \mid k-1}\right]^{-1}=S_{k-1 \mid k-1} S_{k-1 \mid k-1}^{T}
$$

where $Y_{k-1 \mid k-1}$ is information matrix. $S_{k-1 \mid k-1}$ can be obtained by factorizing the inverse information matrix, which is used to evaluate the cubature points.

Then the cubature point can be obtained as follows:

$$
\begin{aligned}
& X_{i, k-1 \mid k-1}=S_{k-1 \mid k-1} \xi_{i}+\widehat{x}_{k-1 \mid k-1}, \\
& X_{i, k-1 \mid k-1}^{*}=f\left(X_{i, k-1 \mid k-1}, u_{k-1}\right),
\end{aligned}
$$

where $\left\{\xi_{i}\right\}$ is the matrix with a set of unit vector as shown in

$$
\sqrt{n}\left\{\left(\begin{array}{c}
1 \\
0 \\
\vdots \\
0
\end{array}\right), \ldots,\left(\begin{array}{c}
0 \\
\vdots \\
0 \\
1
\end{array}\right),\left(\begin{array}{c}
-1 \\
0 \\
\vdots \\
0
\end{array}\right), \ldots,\left(\begin{array}{c}
0 \\
\vdots \\
0 \\
-1
\end{array}\right)\right\}
$$

The predicted state and predicted error covariance can be obtained based on CKF:

$$
\begin{aligned}
\widehat{x}_{k \mid k-1}= & \frac{1}{2 n} \sum_{i=1}^{2 n} X_{i, k-1 \mid k-1}^{*}, \\
P_{k \mid k-1}= & \frac{1}{2 n} \sum_{i=1}^{2 n} X_{i, k-1 \mid k-1}^{*} X_{i, k-1 \mid k-1}^{* T}-\widehat{x}_{k \mid k-1} \widehat{x}_{k \mid k-1}^{T} \\
& +Q_{k-1},
\end{aligned}
$$

From (4), the predicted information matrix and the predicted information state vector can be derived:

$$
\begin{aligned}
Y_{k \mid k-1} & =P_{k \mid k-1}^{-1}, \\
y_{k \mid k-1} & =P_{k \mid k-1}^{-1} \widehat{x}_{k \mid k-1}=Y_{k \mid k-1} \widehat{x}_{k \mid k-1} \\
& =\frac{1}{2 n}\left[Y_{k \mid k-1} \sum_{i=1}^{2 n} X_{i, k-1 \mid k-1}^{*}\right] .
\end{aligned}
$$

\subsection{Measurement Update. Consider}

$$
\left[Y_{k \mid k-1}\right]^{-1}=S_{k \mid k-1} S_{k \mid k-1}^{T},
$$

where $S_{k \mid k-1}$ can be obtained by factorizing the inverse predicted matrix $Y_{k \mid k-1}$.

The cubature points are evaluated as

$$
\chi_{i, k \mid k-1}=S_{k \mid k-1} \xi_{i}+\widehat{x}_{k \mid k-1} .
$$

The propagated cubature points are evaluated as

$$
Z_{i, k \mid k-1}=h\left(\chi_{i, k \mid k-1}\right) .
$$

The predicted measurement is estimated:

$$
\widehat{z}_{k \mid k-1}=\frac{1}{2 n} \sum_{i=1}^{2 n} Z_{i, k \mid k-1}
$$

The cross-covariance matrix is estimated:

$$
P_{x z, k \mid k-1}=\frac{1}{2 n} \sum_{i=1}^{2 n} \chi_{i, k \mid k-1} Z_{i, k \mid k-1}^{T}-\widehat{x}_{k \mid k-1} \widehat{z}_{k \mid k-1}^{T} .
$$

Then information state distribution and information matrix can be obtained from (5) and (11):

$$
\begin{aligned}
& i_{k}=Y_{k \mid k-1} P_{x z, k \mid k-1} R_{k}^{-1}\left[v_{k}+P_{x z, k \mid k-1}^{T} Y_{k \mid k-1}^{T} \widehat{x}_{k \mid k-1}\right] \\
& I_{k}=Y_{k \mid k-1} P_{x z, k \mid k-1} R_{k}^{-1} P_{x z, k \mid k-1}^{T} Y_{k \mid k-1}^{T},
\end{aligned}
$$

where $v_{k}=z-\widehat{z}_{k \mid k-1}$.

From (5) and (12), the updated information matrix and updated information can be given, respectively:

$$
\begin{aligned}
& Y_{k \mid k}=Y_{k \mid k-1}+I_{k}, \\
& y_{k \mid k}=y_{k \mid k-1}+i_{k} .
\end{aligned}
$$

Then the estimated state vector $\widehat{x}_{k \mid k}$ and estimated error covariance $P_{k \mid k}$ can be derived as follows:

$$
\begin{aligned}
& \widehat{x}_{k \mid k}=Y_{k \mid k}{ }^{-1} y_{k \mid k}, \\
& P_{k \mid k}=Y_{k \mid k}{ }^{-1} I_{n},
\end{aligned}
$$

where $I_{n}$ is the $n$ dimensional unit matrix. 


\section{Interacting Multiple Models Cubature Information Filtering Algorithm}

As the information filter can be extended straight to design multisensory fusion algorithm, in this paper, the proposed IMMCIF includes the merits of CIF algorithm and IMM algorithm. The main factor of IMMCIF is that it calculates the state distribution and error covariance matrix by choosing the cubature points with equal weight and then processes the filter update by calculating the information vector and information matrix. The IMMCIF algorithm includes input integration, CIF, model probability update, and output integration. The structure diagram is shown as in Figure 1.

The detailed filtering processes are as follows.

3.1. Input Integration. Consider

$$
\begin{aligned}
u_{k-1 \mid k-1}^{i / j} & =\frac{p_{i j} u_{k-1}^{i}}{C_{j}}, \\
\widehat{X}_{k-1 \mid k-1}^{0 j} & =\sum \widehat{X}_{k-1 \mid k-1}^{i} u_{k-1 \mid k-1}^{i / j}, \\
P_{k-1 \mid k-1}^{0 j} & =\sum_{i=1}^{r} u_{k-1 \mid k-1}^{i / j}\left\{P_{k-1 \mid k-1}^{i}\right. \\
+ & {\left.\left[\widehat{X}_{k-1 \mid k-1}^{i}-\widehat{X}_{k-1 \mid k-1}^{0 j}\right]\left[\widehat{X}_{k-1 \mid k-1}^{i}-\widehat{X}_{k-1 \mid k-1}^{0 j}\right]^{T}\right\}, }
\end{aligned}
$$

where $C_{j}=\sum_{i=1}^{r} p_{i j} u_{k-1}^{i}, u_{k-1 \mid k-1}^{i / j}$ is the conditional probability of model $i$ at $k-1, u_{k-1}^{i}$ is the probability of model $i$ at $k-1, \widehat{X}_{k-1 \mid k-1}^{0 j}$ is the initial mean value of model $j, P_{k-1 \mid k-1}^{0 j}$ is the initial error covariance, $\widehat{X}_{k-1 \mid k-1}^{i}$ is the estimated value of model $i$ at $k-1$, and $P_{k-1 \mid k-1}^{i}$ is the relative covariance.

3.2. Cubature Information Filtering. Let the mixed initial value and the measure value $(z)$ as the input of each filter at $k$. Then new state vector $\widehat{X}_{k \mid k}^{j}$, the error covariance $P_{k \mid k}^{j}$, the predicted measured value $z_{k \mid k-1}^{j}$, and the residual $v_{k}^{j}$ can be obtained from the CIF which have been derived in Section 2.

The likelihood value $L_{k}^{j}$ is

$$
\begin{aligned}
L_{k}^{j}= & N\left(z ; z_{k \mid k-1}^{j}, v_{k}^{j}\right) \\
= & \frac{1}{\sqrt{2 \pi V_{k}^{j}}} \\
& \cdot \exp \left(-\frac{1}{2}\left[z_{k}-\widehat{z}_{k \mid k-1}^{j}\right]^{T}\left(V_{k}^{j}\right)^{-1}\left[z_{k}-\widehat{z}_{k \mid k-1}^{j}\right]\right),
\end{aligned}
$$

where $V_{k}^{j}$ is the associated covariance of residual $v_{k}^{j}$.

3.3. Model Probability Update. Bayesian hypothesis testing method is used to evaluate the model probability and calculate the residual of each filter. It is known that once the filter model matches with the actual model, the filtering residual

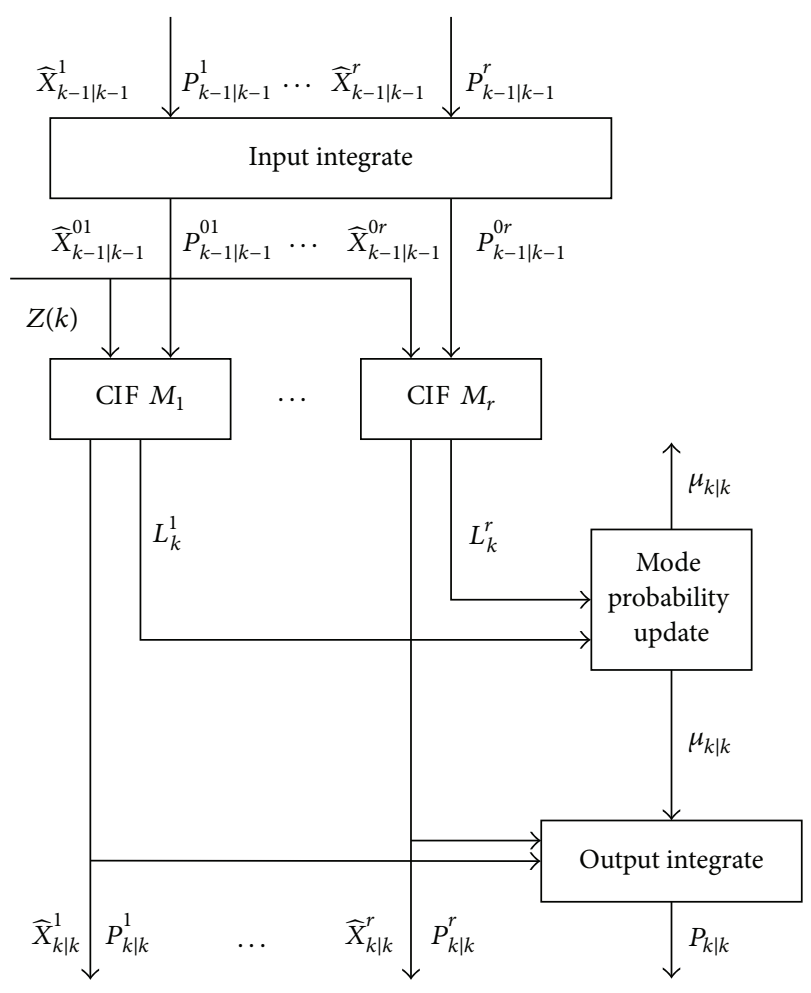

Figure 1: IMMCIF structure diagram.

is zero mean, and the variance $v(k)$ is Gaussian White Noise. Therefore from the likelihood function of model matching $m_{i}$ at $k$, the model probability can be updated from

$$
u_{k}^{j}=\frac{L_{k}^{j} C_{j}}{\sum_{j=1}^{n_{m}} L_{k}^{j} C_{j}} .
$$

3.4. Output Integration. The probabilities of model are fused with the estimated value of each filter based on the given weights; then the final output of the IMMCIF is calculated as

$$
\begin{aligned}
\widehat{X}_{k \mid k} & =\sum_{j=1}^{r} X_{k \mid k}^{j} u_{k}^{j}, \\
P_{k \mid k} & =\sum_{j=1}^{r} u_{k}^{j}\left\{P_{k \mid k}^{j}+\left[\widehat{X}_{k \mid k}^{j}-\widehat{X}_{k \mid k}\right]\left[\widehat{X}_{k \mid k}^{j}-\widehat{X}_{k \mid k}\right]^{T}\right\} .
\end{aligned}
$$

\section{Results and Discussion}

In this section, we consider a classical target tracking scenario where the target trajectory is shown in Figure 2. Let the state vector at time $k$ be $X_{k}=[x, \dot{x}, y, \dot{y}]^{T}$, where $x$ and $y$ are the position variable in $X$ - and $Y$-bearing and $\dot{x}$ and $\dot{y}$ are the velocity variable in $X$ - and $Y$-bearing. 


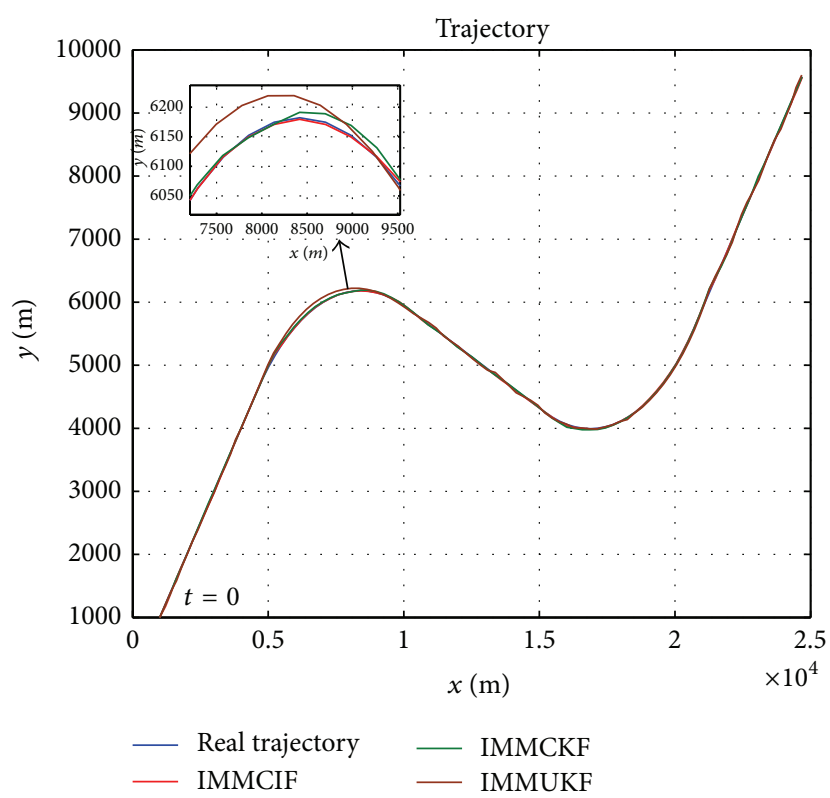

FIgURE 2: Target trajectory.

The CT (coordinated turn) model is

$$
F 2=\left[\begin{array}{cccc}
1 & \frac{\sin (\omega T)}{\omega} & 0 & \frac{(\cos (\omega T)-1)}{\omega} \\
0 & \cos (\omega T) & 0 & -\sin (\omega T) \\
0 & \frac{(1-\cos (\omega T))}{\omega} & 1 & \frac{\sin (\omega T)}{\omega} \\
0 & \sin (\omega T) & 0 & \cos (\omega T)
\end{array}\right],
$$

where $\omega$ is the turn rate of the target and $T$ is the sampling interval.

The right turn rate is defined as $-3^{\circ}$, and the left turn rate is defined as $3^{\circ}$.

The measure equation of the system is

$$
Z=\left[\begin{array}{llll}
1 & 0 & 0 & 0 \\
0 & 0 & 1 & 0
\end{array}\right]+R
$$

where $R$ is the measurement noise of the system.

The initial state is $X_{0}=[1000 \mathrm{~m}, 200 \mathrm{~m} / \mathrm{s}, 1000 \mathrm{~m}$, $200 \mathrm{~m} / \mathrm{s}]^{T}$, and the initial associate covariance is $P_{0}=$ $\operatorname{diag}([1000,10,1000,10])$, the process noise $Q \sim N(0, q)$, with $q=[10,0 ; 0,10]$, and the process noise weight matrix is $G=\left[T^{2} / 2,0 ; T, 0 ; 0, T^{2} / 2 ; 0, T\right]$. The measurement noise $R \sim N(0, r)$, with $r=\operatorname{diag}([200,0.1])$. The simulation time is $\operatorname{sim}$ Time $=100 \mathrm{~s}$, and the step time $T=1 \mathrm{~s}$. The target turns right during $20 \mathrm{~s} \sim 40 \mathrm{~s}$, turns left during $60 \mathrm{~s} \sim 80 \mathrm{~s}$, and maintains uniform motion during the other time. The model transition probability is

$$
p_{i j}=\left[\begin{array}{ccc}
0.9 & 0.05 & 0.05 \\
0.1 & 0.8 & 0.1 \\
0.05 & 0.15 & 0.8
\end{array}\right] \text {. }
$$

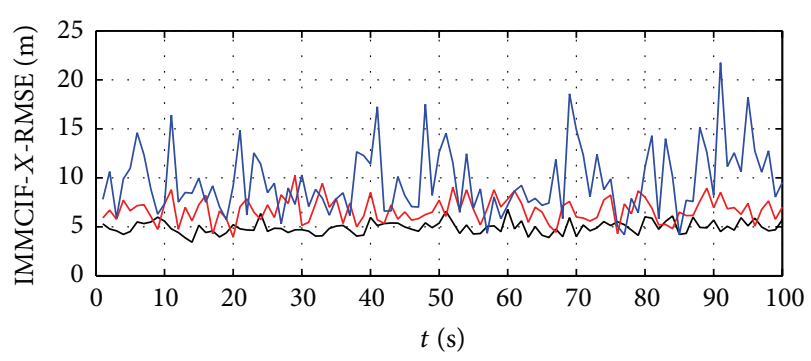

(a)

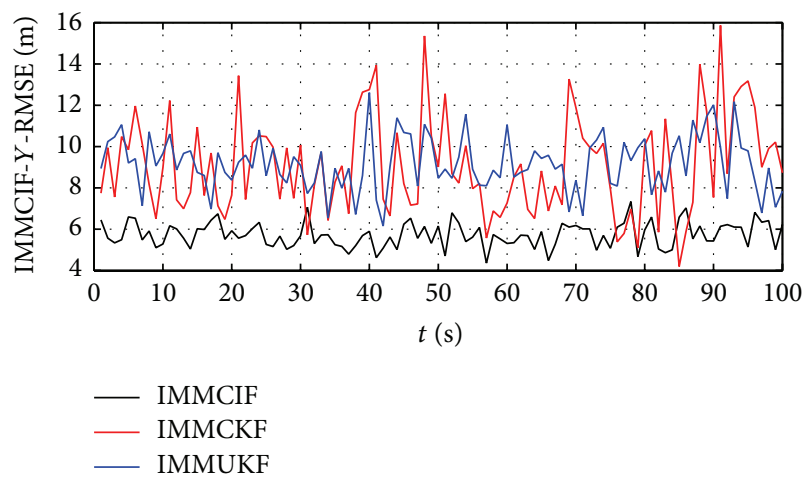

(b)

FIGURE 3: RMSEs of (a) $X$-position and (b) $Y$-position.

TABLE 1: The RMSEs of IMMCIF, IMMCKF, and IMMUKF.

\begin{tabular}{lccc}
\hline & IMMCIF & IMMCKF & IMMUKF \\
\hline RMSE_ $X(\mathrm{~m})$ & 4.9371 & 6.6262 & 9.6817 \\
RMSE_X_V $(\mathrm{m} / \mathrm{s})$ & 2.8419 & 3.2512 & 4.2409 \\
RMSE_$Y(\mathrm{~m})$ & 5.7319 & 9.0982 & 9.1862 \\
RMSE_$Y \_V(\mathrm{~m} / \mathrm{s})$ & 4.5933 & 5.6901 & 5.8375 \\
\hline
\end{tabular}

The root-mean square error (RMSE) of position and velocity is used to compare the performance of two filtering algorithms. The RMSE defined in state vector $X$ at $k$ is

$$
\mathrm{RMSE}=\sqrt{\frac{\sum_{n=1}^{n}(1 / k) \sum_{k=1}^{k}\left(X_{k, n}-\widehat{X}_{k, n}\right)^{2}}{n}},
$$

where $X_{k, n}$ is the real state vector of $n$ Monte Carlo simulation and $\widehat{X}_{k, n}$ is the estimated state vector of $n$ Monte Carlo simulation. The simulation results are shown in Figures 2, 3, and 4.

Figures 2, 3, and 4 and Table 1 show the contrast of the tracking accuracy with IMMCIF, IMMCKF, and IMMUKF. It can be seen clearly from the figures that IMMCIF does better in tracking precision than IMMCKF and IMMUKF, while all of them exhibit stable characteristics and there is no error divergence.

Figures 5(a), 5(b), and 5(c) show the mode probabilities of IMMCIF, IMMCKF, and IMMUKF, which demonstrate that all of them can effectively track the target maneuvering characteristics at preliminary stage. And the mode probabilities correspond well to the maneuvering target, which will 

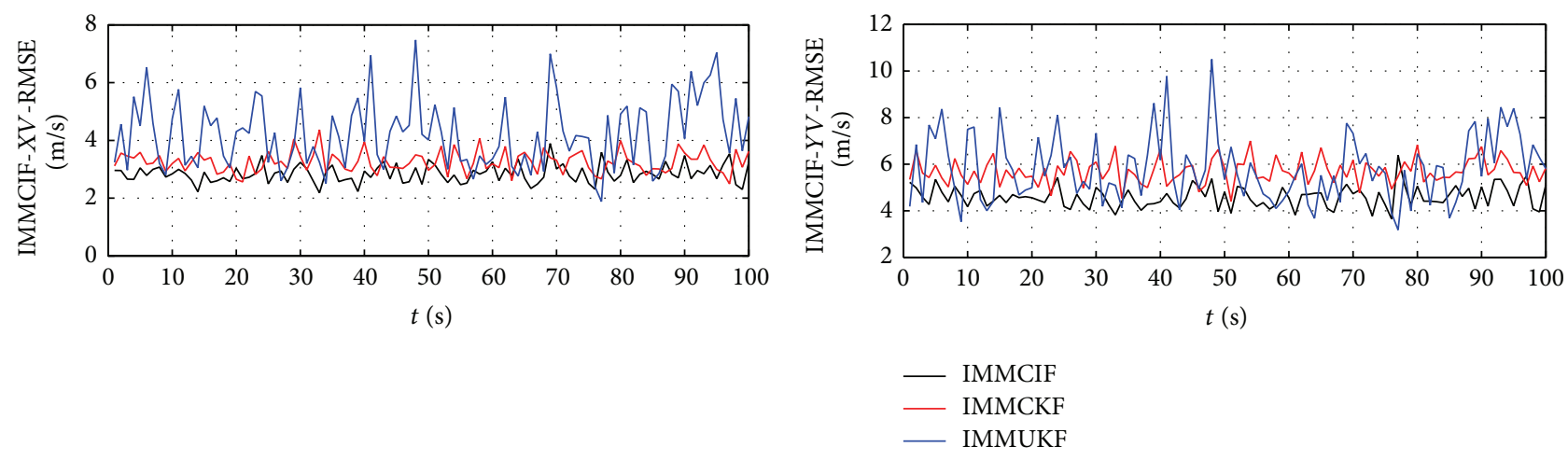

(a)

(b)

FIGURE 4: RMSEs of (a) $X$-velocity and (b) $Y$-velocity.
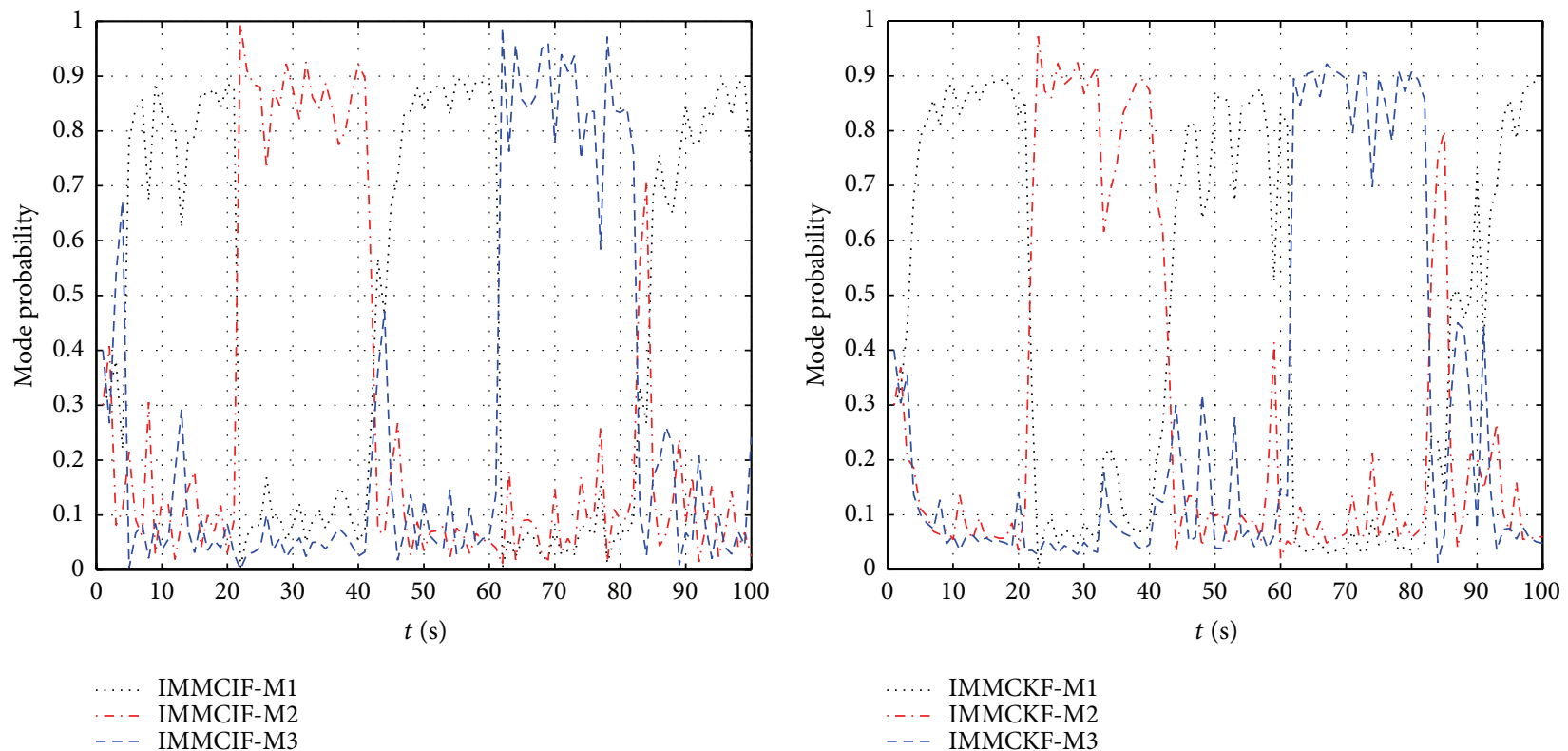

(a) IMMCIF

(b) IMMCKF

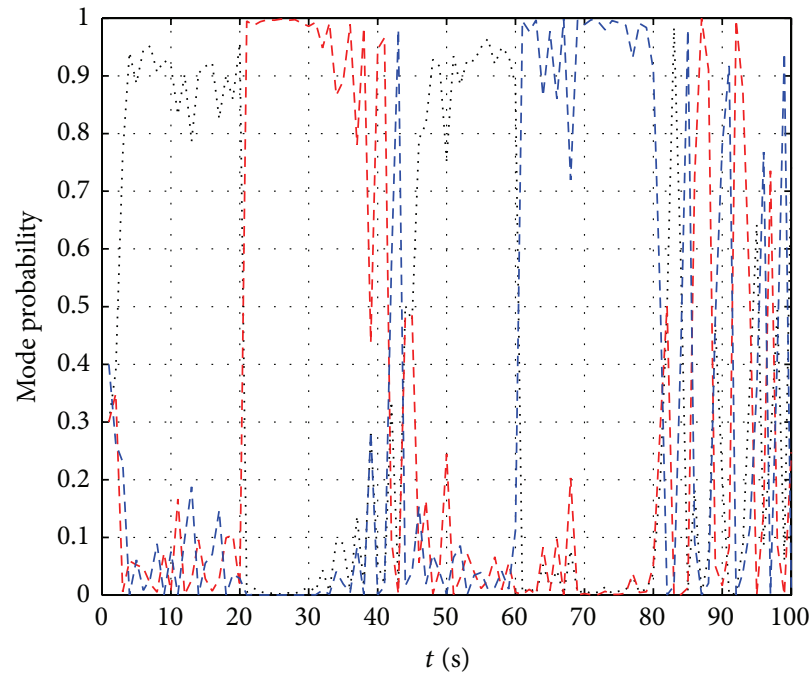

$\begin{array}{ll} & \\ \ldots . & \text { IMMUKF-M } \\ \ldots- & \text { IMMUKF-M2 }\end{array}$

- - - IMMUKF-M3

(c) IMMUKF

Figure 5: Mode probabilities of IMMs. 


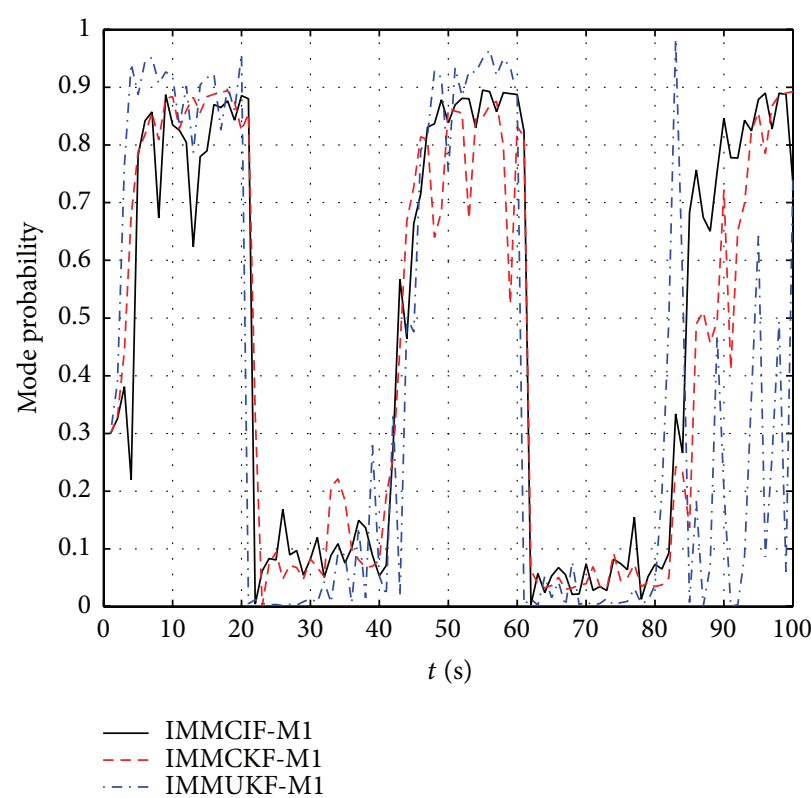

(a) Mode 1

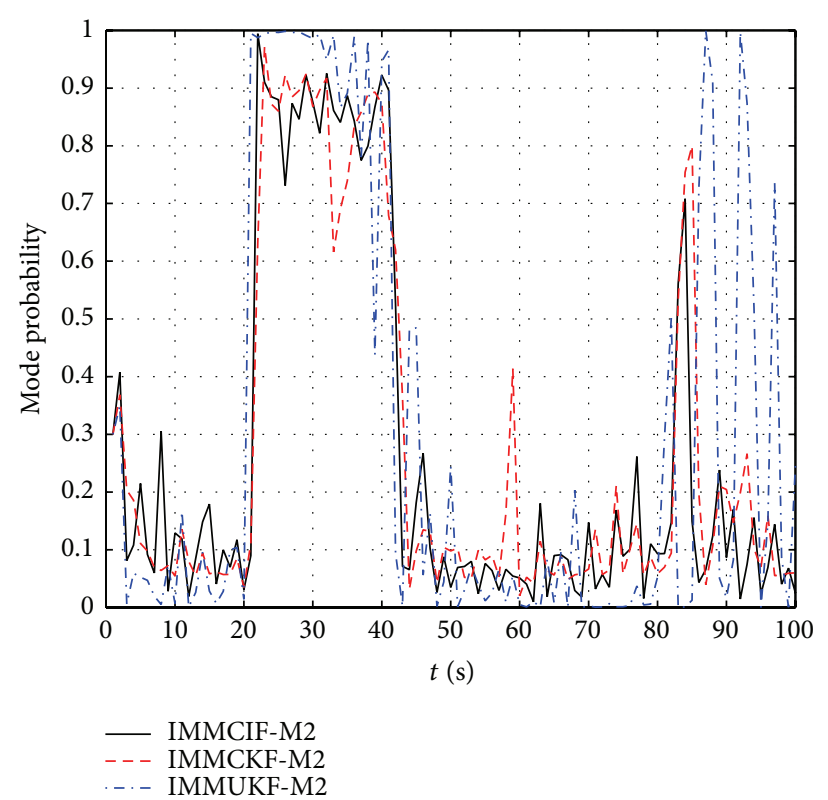

(b) Mode 2

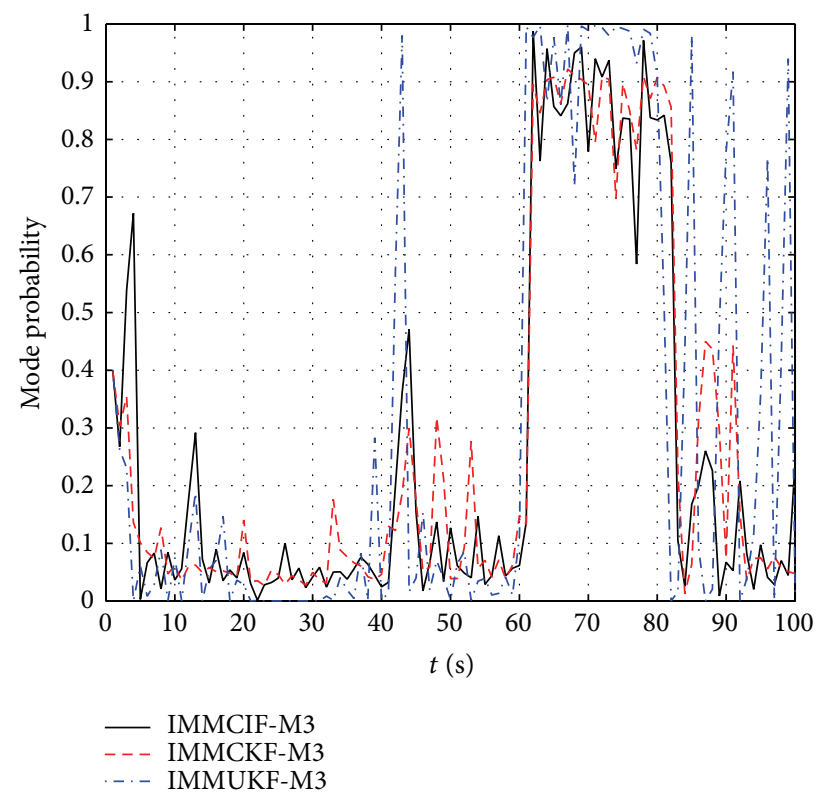

(c) Mode 3

FIGURE 6: Comparisons of mode probability of IMMs.

change when motion state transforms from CV trajectory to CT trajectory and vice versa. Mode 1 is constant velocity (CV) mode, Mode 2 is right coordinate turn (CT) mode, and Mode 3 is left coordinate turn (CT) mode.

The comparisons of mode probabilities of IMMCIF, IMMCKF, and IMMUKF are shown in Figures 6(a), 6(b), and 6(c). The figures show that the IMMCIF, IMMCKF, and IMMUKF can capture the kinematics of maneuvering in time once the motion state changes when $t=20 \mathrm{~s}, t=40 \mathrm{~s}$, $t=60 \mathrm{~s}$, and $t=80 \mathrm{~s}$. However as time goes by, IMMCKF and IMMUKF cannot track the maneuvering target well while the IMMCIF is more stable.

\section{Conclusion}

In this paper, the Interacting Multiple Models Cubature Information Filtering (IMMCIF) algorithm is proposed to enhance the precision and quick response of nonlinear maneuvering target tracking problem. This algorithm introduces Cubature Information Filter based on Interacting Multiple Models, which disposes all the models simultaneously through Markov Chain. And it evaluates the information vector and information matrix instead of the state vector and covariance to deal with nonlinear filtering. The simulation results show IMMCIF outperforms IMMCKF and IMMUKF 
which exhibits potential applications in nonlinear target tracking.

\section{Conflict of Interests}

The authors declare that there is no conflict of interests regarding the publication of this paper.

\section{Acknowledgments}

The authors would like to thank all the reviewers for improving the clarity of the presentation of this paper. This work is supported by the China Postdoctoral Science Foundation Grant (2014M550182), Heilongjiang Postdoctoral Special Fund (LBH-TZ0410), and Innovation of Science and Technology Talents in Harbin (2013RFXXJ016).

\section{References}

[1] M. S. Arulampalam, B. Ristic, N. Gordon, and T. Mansell, "Bearings-only tracking of manoeuvring targets using particle filters," EURASIP Journal on Applied Signal Processing, vol. 2004, no. 15, pp. 2351-2365, 2004.

[2] D. F. Bizup and D. E. Brown, "Maneuver detection using the radar range rate measurement," IEEE Transactions on Aerospace and Electronic Systems, vol. 40, no. 1, pp. 330-336, 2004.

[3] K. Jo, K. Chu, and M. Sunwoo, "Interacting multiple model filter-based sensor fusion of GPS with in-vehicle sensors for real-time vehicle positioning," IEEE Transactions on Intelligent Transportation Systems, vol. 13, no. 1, pp. 329-343, 2012.

[4] R. Toledo-Moreo, M. A. Zamora-Izquierdo, B. Úbeda-Miñarro, and A. F. Gómez-Skarmeta, "High-integrity IMM-EKF-based road vehicle navigation with low-cost GPS/SBAS/INS," IEEE Transactions on Intelligent Transportation Systems, vol. 8, no. 3, pp. 491-511, 2007.

[5] L.-Q. Li and W.-X. Xie, "Bearings-only maneuvering target tracking based on fuzzy clustering in a cluttered environment," International Journal of Electronics and Communications (AEÜ), vol. 68 , no. 2, pp. 130-137, 2014.

[6] W. Li, Y. Jia, J. Du, and J. Zhang, "Distributed multiple-model estimation for simultaneous localization and tracking with NLOS mitigation," IEEE Transactions on Vehicular Technology, vol. 62, no. 6, pp. 2824-2830, 2013.

[7] J. Hu, L. Xie, J. Xu, and Z. Xu, "TDOA-based adaptive sensing in multi-agent cooperative target tracking," Signal Processing, vol. 98, pp. 186-196, 2014.

[8] H. A. P. Blom and Y. Bar-Shalom, "The interacting multiple model algorithm for systems with Markovian switching coefficients," IEEE Transactions on Automatic Control, vol. 33, no. 8, pp. 780-783, 1988.

[9] R. Schutz, B. Engelberg, W. Soper, and R. Mottl, "IMM modeling for AEW applications," in Signal and Data Processing of Small Targets 2001, vol. 4473 of Proceedings of SPIE, pp. 210-221, August 2001.

[10] N. Cui, L. Hong, and J. R. Layne, "A comparison of nonlinear filtering approaches with an application to ground target tracking," Signal Processing, vol. 85, no. 8, pp. 1469-1492, 2005.

[11] M. N. Petsios, E. G. Alivizatos, and N. K. Uzunoglu, "Manoeuvring target tracking using multiple bistatic range and rangerate measurements," Signal Processing, vol. 87, no. 4, pp. 665686, 2007.
[12] S. Zhao, Z. Wang, and F. Liu, "State estimation for nonlinear discrete-time systems with Markov jumps and nonhomogeneous transition probabilities," Mathematical Problems in Engineering, vol. 2013, Article ID 293456, 7 pages, 2013.

[13] N. Tudoroiu, M. Zaheeruddin, V. Cretu, and E.-R. Tudoroiu, "IMM-UKF versus frequency analysis," IEEE Industrial Electronics Magazine, vol. 4, no. 3, pp. 7-18, 2010.

[14] W. Li and Y. Jia, "Location of mobile station with maneuvers using an IMM-based cubature Kalman filter," IEEE Transactions on Industrial Electronics, vol. 59, no. 11, pp. 4338-4348, 2012.

[15] S. Julier, J. Uhlmann, and H. F. Durrant-Whyte, "A new method for the nonlinear transformation of means and covariances in filters and estimators," IEEE Transactions on Automatic Control, vol. 45 , no. 3, pp. 477-482, 2000.

[16] I. Arasaratnam and S. Haykin, "Cubature Kalman filters," IEEE Transactions on Automatic Control, vol. 54, no. 6, pp. 1254-1269, 2009.

[17] K. Pakki, B. Chandra, D.-W. Gu, and I. Postlethwaite, "Cubature information filter and its applications," in Proceedings of the American Control Conference, pp. 3609-3614, San Francisco, Calif, USA, June 2011.

[18] Q. Ge, D. Xu, and C. Wen, "Cubature information filters with correlated noises and their applications in decentralized fusion," Signal Processing, vol. 94, no. 1, pp. 434-444, 2014. 


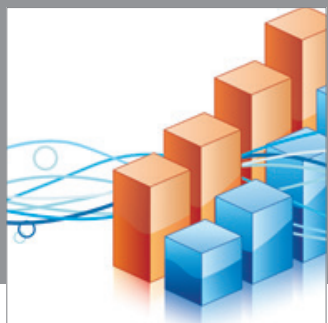

Advances in

Operations Research

mansans

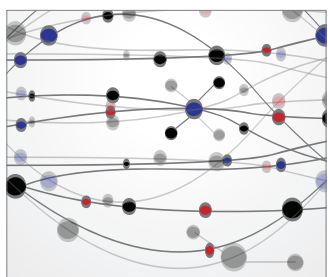

The Scientific World Journal
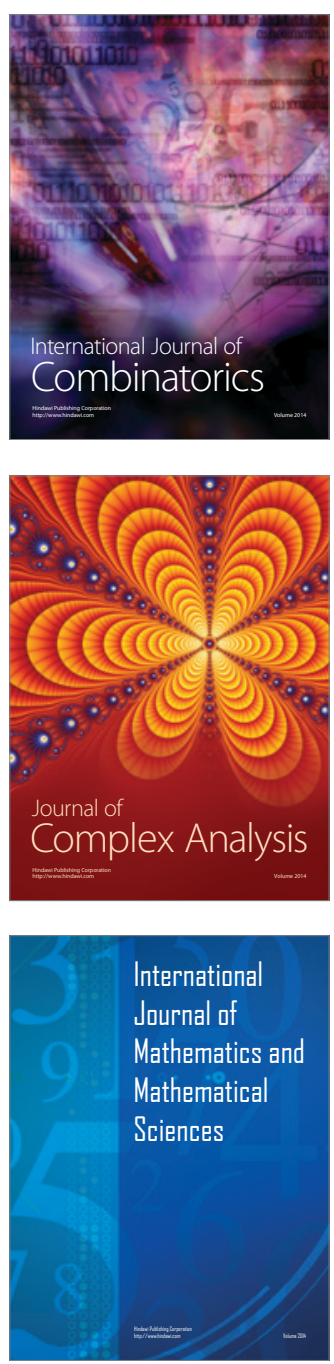
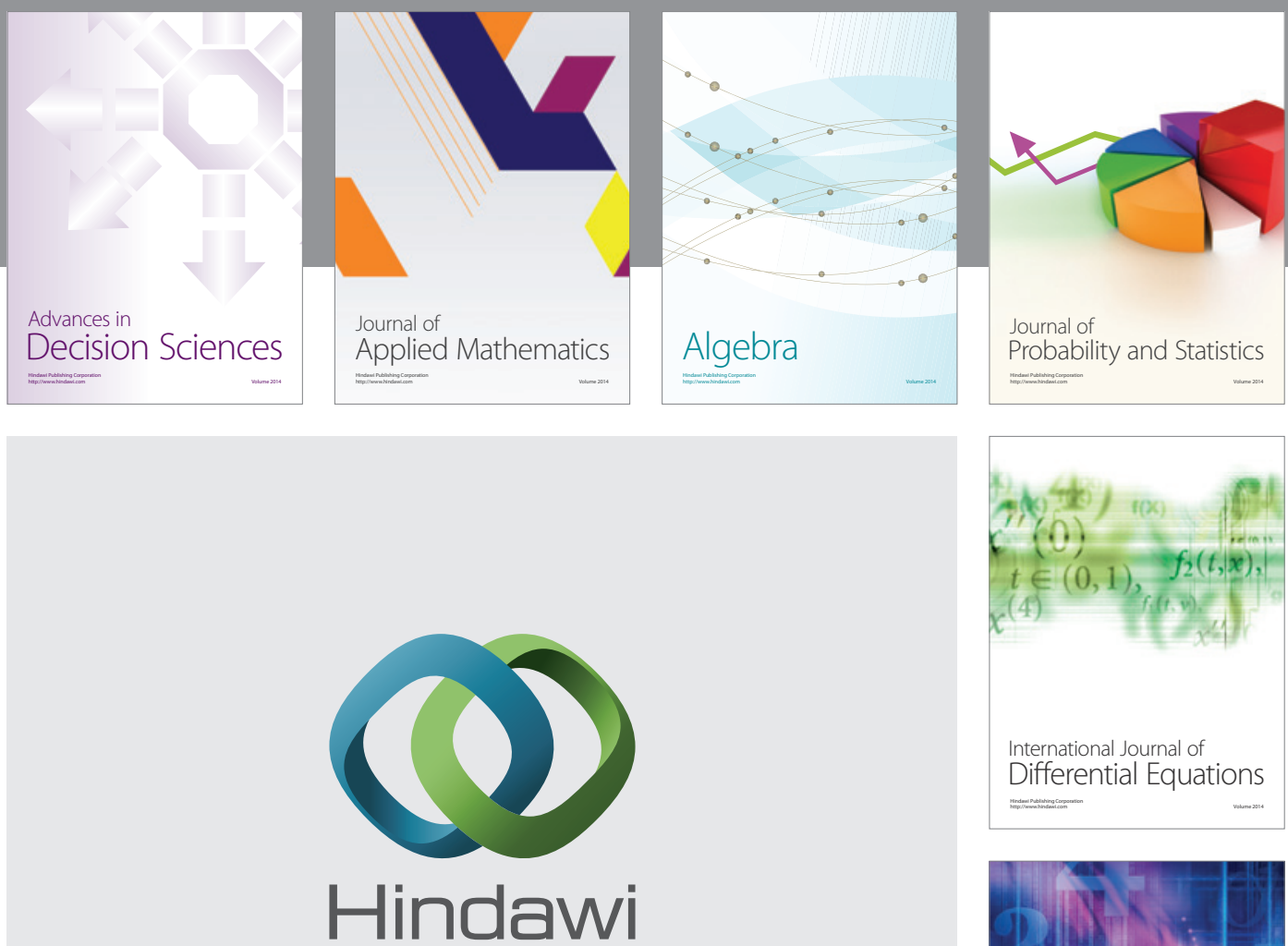

Submit your manuscripts at http://www.hindawi.com
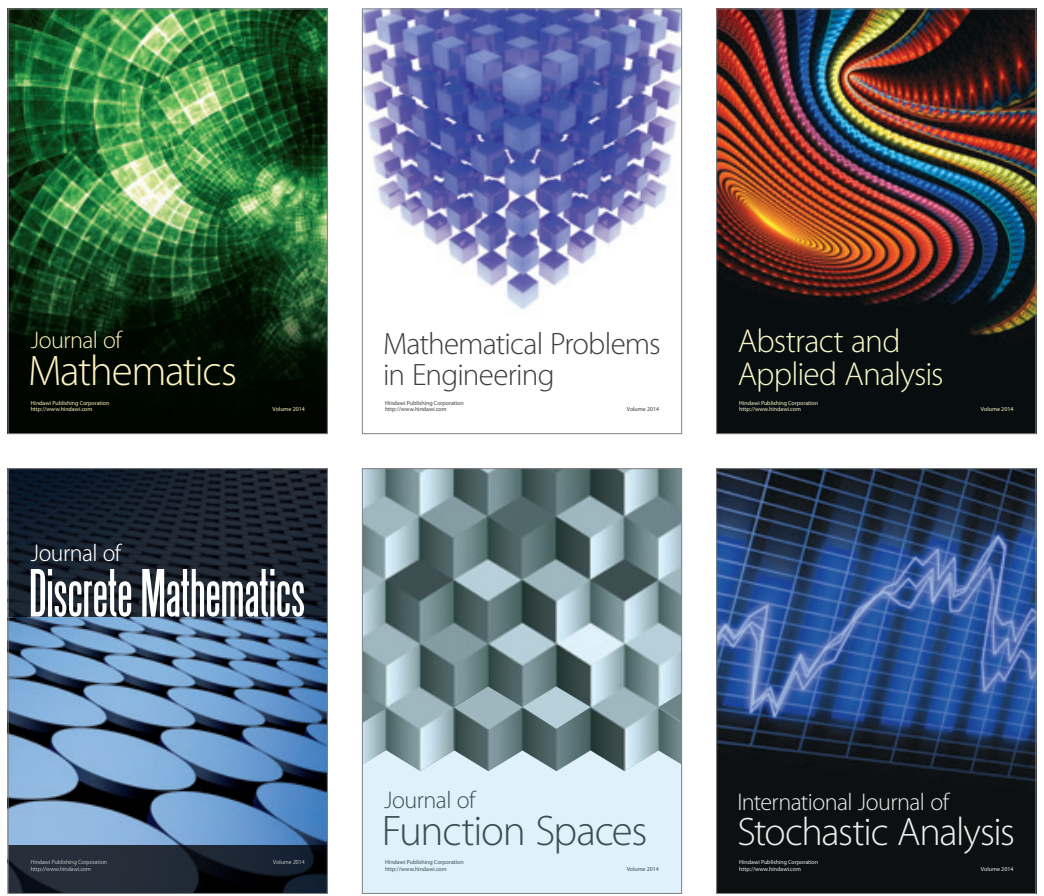

Journal of

Function Spaces

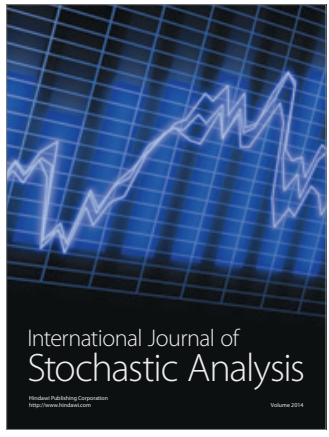

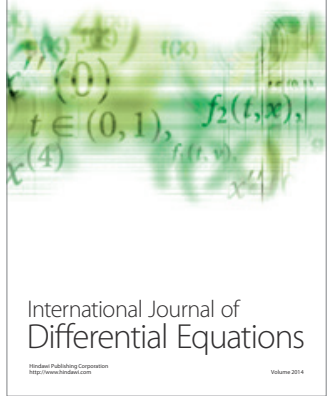
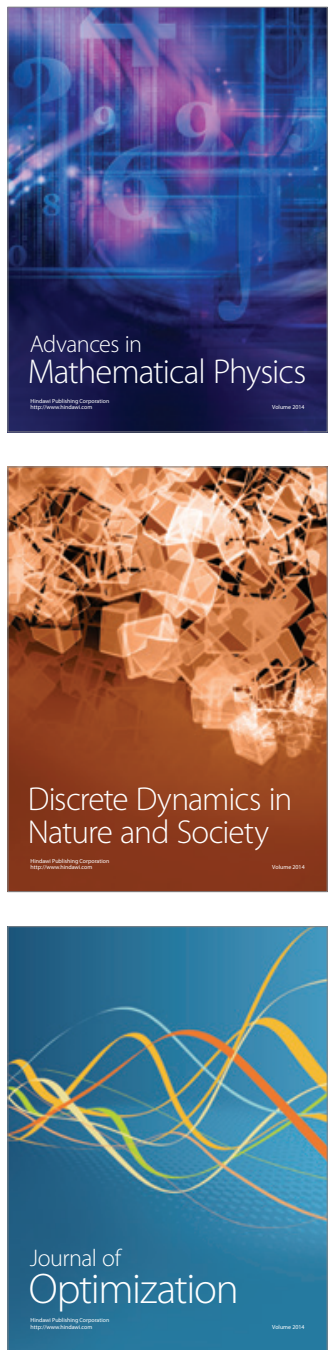